\title{
TRANSFORMATIONS OF SURFACES OF VOSS*
}

\author{
BY \\ LUTHER PFAHLER EISENHART
}

INTRODUCTION

Voss $\nmid$ was the first to study surfaces which are characterized by the property of containing two families of geodesics which form a conjugate system. These surfaces belong to the class admitting a continuous deformation in which a conjugate system remains conjugate; for a surface of Voss it is the geodesic conjugate system which possesses this property. These surfaces play an important rôle also in the determination of congruences whose developables meet the focal surfaces in their lines of curvature. It is the purpose of this paper to establish transformations of surfaces of Voss into surfaces of the same kind, and to study some of the geometrical properties of these transformations.

The geodesic conjugate system of a surface $V$ has the same spherical representation as the asymptotic lines of a pseudospherical surface $P$. Moreover, the determination of all surfaces $V$ with the spherical representation of a given surface $P$ requires the integration of an equation of Laplace with equal invariants. We shall say that each of these surfaces is conjugate to $P$. In $\$ 1$ we recall the formulas defining a pseudospherical surface $P$, the formulas for a conjugate surface $V$ and the equations of a Bäcklund transformation of $P$ into another pseudospherical surface $P_{1}$. Such a transformation involves a constant $\sigma$ (the angle between the tangent planes to $P$ and $P_{1}$ ) and a func$\operatorname{tion} \theta$ whose determination requires the solution of a Riccati equation. When a surface $V$ is given, each pair of quantities $\theta$ and $\sigma$ determine a new surface $V_{1}$ such that the developables of the congruence of lines joining corresponding points on $V$ and $V_{1}$ meet these surfaces in geodesic conjugate systems. Moreover, $V_{1}$ is a conjugate of the surface $P_{1}$ which is determined by the Bäcklund transformation of $P$ by means of $\theta$ and $\sigma$. In order to put in evidence the functions we say that $V_{1}$ is obtained from $V$ by a transformation $\Omega(\theta, \sigma)$.

\footnotetext{
* Presented to the Society, December 31, 1913.

† Ueber diejenigen Flachen auf denen zwei Scharen geodätischer Linien ein conjugirtes System bilden, Sitzung s berichteder K. Akademiezu München (1888), pp. 95102.
}

$\ddagger$ Throughout the paper we shall denote by $V$ a surface of Voss. 
Each surface $V$ conjugate to $P$ is transformable by the same pair of functions $\theta$ and $\sigma$. However, for a given pair $(\theta, \sigma)$ there exists a surface $V$ such that the lines joining its points to the corresponding points on the surface $V_{1}$ resulting from the transformation $\Omega(\theta, \sigma)$ are concurrent. By means of certain additional functions connected with this special case we are able to show that the transformations $\Omega(\theta, \sigma)$ are of the Moutard type for equations with equal invariants. In a subsequent paper we shall show that there exist transformations of general conjugate systems with equal tangential invariants - transformations which are, in certain respects, generalizations of the transformations $\Omega(\theta, \sigma)$.

The transformations $\Omega(\theta, \sigma)$ admit a "theorem of permutability" (cf. $\S 6$ ). The remainder of the paper deals with the congruence of lines joining corresponding points on $V$ and $V_{1}$, and also the other congruence formed by the lines of intersection of the tangent planes at corresponding points on $V$ and $V_{1}$; the latter is a normal congruence.

\section{Equations of a Surface $V$ and Preliminary Formulas}

We consider a surface $V$ referred to the geodesic conjugate system. Since this system has the same spherical representation as the asymptotic lines on a pseudospherical surface $P$, the linear element of this spherical representation can be given the form

$$
d \sigma^{2}=d u^{2}+2 \cos 2 \omega d u d v+d v^{2},
$$

where $\omega$ is a function of $u$ and $v$ satisfying the equation

$$
\frac{\partial^{2} \omega}{\partial u \partial v}+\sin \omega \cos \omega=0 . *
$$

If $X_{1}, Y_{1}, Z_{1}$ and $X_{2}, Y_{2}, Z_{2}$, denote respectively the direction-cosines of the bisectors of the angles between the parametric curves of the spherical representation, and $X, Y, Z$ the direction-cosines of the normal to a surface $V$ with this representation, we have

$$
\frac{\partial X_{1}}{\partial u}=-\frac{\partial \omega}{\partial u} X_{2}-\sin \omega X, \quad \frac{\partial X_{1}}{\partial v}=\frac{\partial \omega}{\partial v} X_{2}+\sin \omega X,
$$

$$
\begin{array}{ll}
\frac{\partial X_{2}}{\partial u}=\frac{\partial \omega}{\partial u} X_{1}-\cos \omega X, & \frac{\partial X_{2}}{\partial v}=-\frac{\partial \omega}{\partial v} X_{1}-\cos \omega X \\
\frac{\partial X}{\partial u}=\sin \omega X_{1}+\cos \omega X_{2}, & \frac{\partial X}{\partial v}=-\sin \omega X_{1}+\cos \omega X_{2} \cdot \dagger
\end{array}
$$

* E., p. 289. A reference of this kind is to the author's Treatise on the differential geometry of curves and surfaces, Ginn and Company, Boston (1909).

† Cf. Bianchi, Lezioni di geometria differenziale, vol. 1, p. 320, Pisa (1902). 
The equation satisfied by the tangential coördinates $X, Y, Z$, and $W$ of the surface $V$ is

$$
\frac{\partial^{2} \phi}{\partial u \partial v}+\cos 2 \omega \phi=0 .^{*}
$$

In terms of the tangential coördinates the rectangular coördinates are of the form

$$
x=W X+\frac{1}{\sin 2 \omega}\left[X_{1} \cos \omega\left(\frac{\partial W}{\partial u}-\frac{\partial W}{\partial v}\right)\right.
$$

$$
\left.+X_{2} \sin \omega\left(\frac{\partial W}{\partial u}+\frac{\partial W}{\partial v}\right)\right] \cdot \dagger
$$

From these we obtain

where

$$
\begin{aligned}
& \frac{\partial x}{\partial u}=-\frac{D}{\sin 2 \omega}\left(\cos \omega X_{1}+\sin \omega X_{2}\right), \\
& \frac{\partial x}{\partial v}=\frac{D^{\prime \prime}}{\sin 2 \omega}\left(\cos \omega X_{1}-\sin \omega X_{2}\right),
\end{aligned}
$$

$$
\begin{aligned}
D & =-\left(\frac{\partial^{2} W}{\partial u^{2}}-2 \cot 2 \omega \frac{\partial \omega}{\partial u} \frac{\partial W}{\partial u}+\frac{2}{\sin 2 \omega} \frac{\partial \omega}{\partial u} \frac{\partial W}{\partial v}+W\right), \\
D^{\prime \prime} & =-\left(\frac{\partial^{2} W}{\partial v^{2}}+\frac{2}{\sin 2 \omega} \frac{\partial \omega}{\partial v} \frac{\partial W}{\partial u}-2 \cot 2 \omega \frac{\partial \omega}{\partial v} \frac{\partial W}{\partial v}+W\right) . \ddagger
\end{aligned}
$$

The Codazzi equations for the surface $V$ may be given the form

$$
\frac{\partial D}{\partial v}-\frac{2}{\sin 2 \omega} \frac{\partial \omega}{\partial u} D^{\prime \prime}=0, \quad \frac{\partial D^{\prime \prime}}{\partial u}-\frac{2}{\sin 2 \omega} \frac{\partial \omega}{\partial v} D=0 . \S
$$

When the pseudospherical surface $P$ with the representation (1) of its asymptotic lines is subjected to a Bäcklund transformation, the linear element of the spherical representation of the asymptotic lines on the new surface $P_{1}$ is given by

$$
d \sigma_{1}^{2}=d u^{2}+2 \cos 2 \theta d u d v+d v^{2},
$$

where $\theta$ is a solution of equation (2) given by

$$
\begin{aligned}
& \sin \sigma\left(\frac{\partial \theta}{\partial u}-\frac{\partial \omega}{\partial u}\right)+(\cos \sigma+1) \sin (\theta+\omega)=0, \\
& \sin \sigma\left(\frac{\partial \theta}{\partial v}+\frac{\partial \omega}{\partial v}\right)+(\cos \sigma-1) \sin (\theta-\omega)=0,
\end{aligned}
$$

\footnotetext{
* E., p. 415.

$\dagger$ E., p. 163 with the aid of equations (3).

$\ddagger$ Cf. E., p. 164.

\&., p. 200 . Equations (36).
} 
$\sigma$ being the constant angle between the tangent planes to $P$ and $P_{1}$ at corresponding points. ${ }^{*}$ The direction-cosines $X^{\prime}, Y^{\prime}$, and $Z^{\prime}$ of the normal to $P_{1}$ are of the form

$$
X^{\prime}=\cos \sigma X+\sin \sigma\left(\sin \theta X_{1}-\cos \theta X_{2}\right), \dagger
$$

and the direction-cosines $\bar{X}, \bar{Y}$, and $\bar{Z}$ of the line joining corresponding points of $P$ and $P_{1}$, and tangent to these surfaces at these points, are of the form

$$
\bar{X}=\cos \theta X_{1}+\sin \theta X_{2}
$$

From (11) we obtain with the aid of equations (3)

$$
\frac{\partial X^{\prime}}{\partial u}=\sin \theta X_{1}^{\prime}+\cos \theta X_{2}^{\prime}, \quad \frac{\partial X^{\prime}}{\partial v}=-\sin \theta X_{1}^{\prime}+\cos \theta X_{2}^{\prime},
$$

where we have put

$$
\begin{aligned}
X_{1}^{\prime}= & \sin \omega\left(\cos \sigma\left(\sin \theta X_{1}-\cos \theta X_{2}\right)-\sin \sigma X\right) \\
& -\cos \omega\left(\cos \theta X_{1}+\sin \theta X_{2}\right), \\
X_{2}^{\prime}=-\cos \omega\left(\cos \sigma\left(\sin \theta X_{1}-\cos \theta X_{2}\right)-\sin \sigma X\right) & -\sin \omega\left(\cos \theta X_{1}+\sin \theta X_{2}\right) .
\end{aligned}
$$

From these follow by differentiation

$$
\begin{array}{ll}
\frac{\partial X_{1}^{\prime}}{\partial u}=-\frac{\partial \theta}{\partial u} X_{2}^{\prime}-\sin \theta X^{\prime}, & \frac{\partial X_{1}^{\prime}}{\partial v}=\frac{\partial \theta}{\partial v} X_{2}^{\prime}+\sin \theta X^{\prime}, \\
\frac{\partial X_{2}^{\prime}}{\partial u}=\frac{\partial \theta}{\partial v} X_{1}^{\prime}-\cos \theta X^{\prime}, & \frac{\partial X_{2}^{\prime}}{\partial v}=-\frac{\partial \theta}{\partial v} X_{1}^{\prime}-\cos \theta X^{\prime},
\end{array}
$$

equations which are analogous to (3). Equations (14) are equivalent to

$\cos \theta X_{1}^{\prime}+\sin \theta X_{2}^{\prime}=-\sin (\theta-\omega)\left[\cos \sigma\left(\sin \theta X_{1}\right.\right.$

$$
\left.\left.-\cos \theta X_{2}\right)-\sin \sigma X\right]-\cos (\theta-\omega)\left(\cos \theta X_{1}+\sin \theta X_{2}\right) \text {, }
$$

$\cos \theta X_{1}^{\prime}-\sin \theta X_{2}^{\prime}=\sin (\theta+\omega)\left[\cos \sigma\left(\sin \theta X_{1}\right.\right.$

$$
\left.\left.-\cos \theta X_{2}\right)-\sin \sigma X\right]-\cos (\theta+\omega)\left(\cos \theta X_{1}+\sin \theta X_{2}\right) \text {. }
$$

2. The Transformation $\Omega(\theta, \sigma)$ of the Surfaces $V$

Each solution $W_{1}$ of the equation

$$
\frac{\partial^{2} \phi_{1}}{\partial u \partial v}+\cos 2 \theta \phi_{1}=0
$$

* Equations (10) may be obtained from equations (44) E., p. 289, by replacing $\cos \sigma$ by $-\cos \sigma$.

$\dagger$ This follows from E., p. 284.

$\ddagger$ E., l. c. 
determines a surface $V$ with the spherical representation (9) of its geodesic conjugate system. The rectangular coördinates of the corresponding surface $V_{1}$ are of the form

$$
\begin{aligned}
& x_{1}=W_{1} X^{\prime}+\frac{1}{\sin 2 \theta}\left[X_{1}^{\prime} \cos \theta\left(\frac{\partial W_{1}}{\partial u}-\frac{\partial W_{1}}{\partial v}\right)\right. \\
& \left.+X_{2}^{\prime} \sin \theta\left(\frac{\partial W_{1}}{\partial u}+\frac{\partial W_{1}}{\partial v}\right)\right]
\end{aligned}
$$

where $X^{\prime}, X_{1}^{\prime}$, and $X_{2}^{\prime}$ have the significance given by equations (11) and (14).

It is our purpose to show that when a surface $V$ whose coördinates are given by (5) is known, it is possible to find a surface $V_{1}$ defined by (18), such that the developables of the congruence formed by the joins of corresponding points of $V$ and $V_{1}$ cut these surfaces in their respective geodesic conjugate systems.

If we write the coördinates of any point on a line of this congruence in the form

$$
x+t\left(x_{1}-x\right), \quad y+t\left(y_{1}-y\right), \quad z+t\left(z_{1}-z\right) .
$$

it must be possible to find two values of $t$, say $t_{1}$ and $t_{2}$, such that

$$
\begin{aligned}
& \frac{\left(1-t_{1}\right) \frac{\partial x}{\partial u}+t_{1} \frac{\partial x_{1}}{\partial u}}{x_{1}-x}=\frac{\left(1-t_{1}\right) \frac{\partial y}{\partial u}+t_{1} \frac{\partial y_{1}}{\partial u}}{y_{1}-y}=\frac{\left(1-t_{1}\right) \frac{\partial z}{\partial u}+t_{1} \frac{\partial z_{1}}{\partial u}}{z_{1}-z}, \\
& \frac{\left(1-t_{2}\right) \frac{\partial x}{\partial v}+t_{2} \frac{\partial x_{1}}{\partial v}}{x_{1}-x}=\frac{\left(1-t_{2}\right) \frac{\partial y}{\partial v}+t_{2} \frac{\partial y_{1}}{\partial v}}{y_{1}-y}=\frac{\left(1-t_{2}\right) \frac{\partial z}{\partial v}+t_{2} \frac{\partial z_{1}}{\partial v}}{z_{1}-z} .
\end{aligned}
$$

The necessary and sufficient condition that these equations be consistent is that the following equations be satisfied:

$$
\left|\begin{array}{ccc}
\frac{\partial x}{\partial u} & \frac{\partial y}{\partial u} & \frac{\partial z}{\partial u} \\
\frac{\partial x_{1}}{\partial u} & \frac{\partial y_{1}}{\partial u} & \frac{\partial z_{1}}{\partial u} \\
x_{1}-x & y_{1}-y & z_{1}-z
\end{array}\right|=0, \quad\left|\begin{array}{ccc}
\frac{\partial x}{\partial v} & \frac{\partial y}{\partial v} & \frac{\partial z}{\partial v} \\
\frac{\partial x_{1}}{\partial v} & \frac{\partial y_{1}}{\partial v} & \frac{\partial z_{1}}{\partial v} \\
x_{1}-x & y_{1}-y & z_{1}-z
\end{array}\right|=0
$$

The equations for $V_{1}$ analogous to (6) are

$$
\begin{aligned}
& \frac{\partial x_{1}}{\partial u}=-\frac{D_{1}}{\sin 2 \theta}\left(\cos \theta X_{1}^{\prime}+\sin \theta X_{2}^{\prime}\right), \\
& \frac{\partial x_{1}}{\partial v}=\frac{D_{1}^{\prime \prime}}{\sin 2 \theta}\left(\cos \theta X_{1}^{\prime}-\sin \theta X_{2}^{\prime}\right) .
\end{aligned}
$$


Again with the aid of equations (11) and (14) we obtain from (5) and (18) the expression

$$
x_{1}-x=A X+B X_{1}+C X_{2} \text {, }
$$

where

$$
\begin{aligned}
A=W_{1} \cos \sigma-W & +\frac{\sin \sigma}{\sin 2 \theta}\left(\sin (\theta-\omega) \frac{\partial W_{1}}{\partial u}+\sin (\theta+\omega) \frac{\partial W_{1}}{\partial v}\right) \\
B=W_{1} \sin \sigma \sin \theta & -\frac{\cos \sigma \sin \theta}{\sin 2 \theta}\left(\sin (\theta-\omega) \frac{\partial W_{1}}{\partial u}\right. \\
& \left.+\sin (\theta+\omega) \frac{\partial W_{1}}{\partial v}\right)-\frac{\cos \omega}{\sin 2 \omega}\left(\frac{\partial W}{\partial u}-\frac{\partial W}{\partial v}\right) \\
& +\frac{\cos \theta}{\sin 2 \theta}\left(\cos (\theta+\omega) \frac{\partial W_{1}}{\partial v}-\cos (\theta-\omega) \frac{\partial W_{1}}{\partial u}\right), \\
C=-W_{1} \sin \sigma \cos \theta & +\frac{\cos \sigma \cos \theta}{\sin 2 \theta}\left(\sin (\theta-\omega) \frac{\partial W_{1}}{\partial u}\right. \\
& \left.+\sin (\theta+\omega) \frac{\partial W_{1}}{\partial v}\right)-\frac{\sin \omega}{\sin 2 \omega}\left(\frac{\partial W}{\partial u}+\frac{\partial W}{\partial v}\right) \\
& +\frac{\sin \theta}{\sin 2 \theta}\left(\cos (\theta+\omega) \frac{\partial W_{1}}{\partial v}-\cos (\theta-\omega) \frac{\partial W_{1}}{\partial u}\right) .
\end{aligned}
$$

When these values are substituted in (21) and use is made of (16) in the reduction, we obtain

$$
\begin{aligned}
& A \cos (\theta-\omega)(\cos \sigma-1)+B \sin \omega \sin \sigma-C \cos \omega \sin \sigma=0, \\
& A \cos (\theta+\omega)(\cos \sigma+1)-B \sin \omega \sin \sigma-C \cos \omega \sin \sigma=0 .
\end{aligned}
$$

In consequence of (24) these equations, and consequently equations (21), are equivalent to

$$
\begin{aligned}
& \frac{\partial W_{1}}{\partial u}+\frac{\cos \sigma+1}{\sin \sigma} \cos (\theta+\omega) W_{1}=-\frac{\partial W}{\partial u}+\frac{1+\cos \sigma}{\sin \sigma} \cos (\theta+\omega) W, \\
& \frac{\partial W_{1}}{\partial v}+\frac{\cos \sigma-1}{\sin \sigma} \cos (\theta-\omega) W_{1}=\frac{\partial W}{\partial v}+\frac{1-\cos \sigma}{\sin \sigma} \cos (\theta-\omega) W .
\end{aligned}
$$

Since $\theta$ satisfies equations (10), the condition of integrability of equations (25) is satisfied, as is readily shown, and furthermore the function $W_{1}$ so defined is a solution of equation (17). We shall find shortly $(\$ 5)$ that the integration of equations (25) requires one quadrature only. Hence we have

TheOREM I. Each solution of equations (10) determines a transformation $\Omega(\theta, \sigma)$ of a given surface $V$ into a surface $V_{1}$ such that the developables of the congruence formed by the joins of corresponding points on $V$ and $V_{1}$ cut these 
surfaces in the geodesic conjugate systems. Moreover, when $\theta$ is known, the further determination of $V_{1}$ requires only a quadrature.

The second fundamental coefficients of $V_{1}$, namely $D_{1}, D_{1}^{\prime \prime}$, are given by expressions similar to (7). In consequence of (25) these can be given the form

$$
\begin{aligned}
& D_{1}=-D-\frac{2 A}{\sin \sigma \sin 2 \omega}\left(2 \cos (\theta-\omega) \frac{\partial \omega}{\partial u}-\frac{\cos \sigma+1}{\sin \sigma} \sin 2 \omega\right), \\
& D_{1}^{\prime \prime}=D^{\prime \prime}-\frac{2 A}{\sin \sigma \sin 2 \omega}\left(2 \cos (\theta+\omega) \frac{\partial \omega}{\partial v}+\frac{1-\cos \sigma}{\sin \sigma} \sin 2 \omega\right) .
\end{aligned}
$$

\section{The Congruence Associated with a Transformation $\Omega(\theta, \sigma)$}

With the aid of (25) the expressions (24) are reducible to

$$
\begin{aligned}
& A=\frac{\sin \sigma}{\sin 2 \theta}\left(\frac{\sin 2 \omega}{\sin \sigma}\left(W_{1}-W \cos \sigma\right)-\sin (\theta-\omega) \frac{\partial W}{\partial u}\right. \\
& B=\frac{A}{\sin \sigma \sin \omega}(\cos \theta \cos \omega-\cos \sigma \sin \theta \sin \omega) . \\
& C=\frac{A}{\sin \sigma \cos \omega}(\cos \theta \cos \omega \cos \sigma-\sin \theta \sin \omega) .
\end{aligned}
$$

From these expressions and (23) it follows that the direction-cosines $\alpha, \beta$, and $\gamma$ of the line joining corresponding points on $V$ and $V_{1}$ are of the form

$$
\begin{aligned}
\alpha \rho=\cos \omega(\cos \theta & \cos \omega-\cos \sigma \sin \omega \sin \theta) X_{1} \\
& +\sin \omega(\cos \theta \cos \omega \cos \sigma-\sin \theta \sin \omega) X_{2} \\
& +\sin \sigma \sin \omega \cos \omega X,
\end{aligned}
$$

where

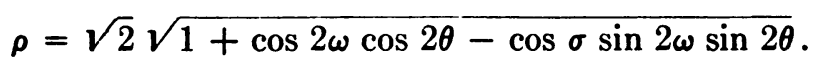

Since the expressions for these direction-cosines involve only the functions of the Bäcklund transformation, we have

Theorem II. If $V$ and $V^{\prime}$ are any two surfaces conjugate to $P$, and $V_{1}$ and $V_{1}^{\prime}$ are obtained by transformations involving the same functions $\theta$ and $\sigma$, the developables of the congruences of lines joining corresponding points of $V$ and $V_{1}$ and of $V^{\prime}$ and $V_{1}^{\prime}$ have the same spherical representation.

In $\$ 4$ we shall find that in certain cases these congruences coincide.

In the tangent plane to $V$ at a point $M$ we draw the line $l$ parallel to the line joining corresponding points of the pseudospherical surfaces $P$ and $P_{1}$ determining a transformation of $V$. The direction-cosines $\bar{X}, \bar{Y}$, and $\bar{Z}$ of 
this line are of the form

$$
\bar{X}=\cos \theta X_{1}+\sin \theta X_{2} .
$$

The direction-cosines $\bar{X}^{\prime}, \bar{Y}^{\prime}$, and $\bar{Z}^{\prime}$ of the line in this plane perpendicular to $l$ are of the form

$$
\bar{X}^{\prime}=-\sin \theta X_{1}+\cos \theta X_{2} \text {. }
$$

With the aid of these expressions equation (28) may be written

$$
\begin{aligned}
2 \alpha \rho= & \sin \sigma \sin 2 \omega X+(\cos 2 \omega+\cos 2 \theta) \bar{X} \\
& +(-\sin 2 \theta+\cos \sigma \sin 2 \omega) X^{\prime} .
\end{aligned}
$$

In view of this expression we are able to give the following construction for the direction of the line through $M$ upon which lies the point $M_{1}$ of the transformed surface $V_{1}$. In the tangent plane to $V$ at $M$ we lay off a unit vector which makes the angle $-2 \theta$ with the line $l$, and in the plane through $l$ and inclined at the angle $\sigma$ to the tangent plane we lay off a unit vector which makes the angle $2 \omega$ with $l$; the vector which is the sum of these two vectors has the desired direction.

It is our purpose now to determine the abscissas of the focal points of the congruence of joins of corresponding points on $V$ and $V_{1}$. To this end we observe that in consequence of $(20)$ we have

$$
\frac{\left(1-t_{1}\right) \sum X^{\prime} \frac{\partial x}{\partial u}}{\sum X^{\prime}\left(x_{1}-x\right)}=\frac{t_{1} \sum X \frac{\partial x_{1}}{\partial u}}{\sum X\left(x_{1}-x\right)}, \quad \frac{\left(1-t_{2}\right) \sum X^{\prime} \frac{\partial x}{\partial v}}{\sum X^{\prime}\left(x_{1}-x\right)}=\frac{t_{2} \sum X \frac{\partial x_{1}}{\partial v}}{\sum X\left(x_{1}-x\right)} .
$$

It is readily shown that

$$
\begin{aligned}
& \sum X \frac{\partial x_{1}}{\partial u}=-\frac{D_{1}}{\sin 2 \theta} \sin \sigma \sin (\theta-\omega), \\
& \sum X \frac{\partial x_{1}}{\partial v}=-\frac{D_{1}^{\prime \prime}}{\sin 2 \theta} \sin \sigma \sin (\theta+\omega), \\
& \sum X^{\prime} \frac{\partial x}{\partial u}=-\frac{D}{\sin 2 \omega} \sin \sigma \sin (\theta-\omega), \\
& \sum X^{\prime} \frac{\partial x}{\partial v}=\frac{D^{\prime \prime}}{\sin 2 \omega} \sin \sigma \sin (\theta+\omega) .
\end{aligned}
$$

In consequence of the values (27) we have also

$$
\sum X^{\prime}\left(x_{1}-x\right)=\frac{\sin 2 \theta}{\sin 2 \omega} A \text {. }
$$

Substituting these expressions in the above equations, we obtain

$$
\left(1-t_{1}\right) D=t_{1} D_{1}, \quad\left(1-t_{2}\right) D^{\prime \prime}=-t_{2} D_{1}^{\prime \prime},
$$


from which we derive the following:

$$
\begin{array}{ll}
t_{1}=\frac{D}{D+D_{1}}, & 1-t_{1}=\frac{D_{1}}{D+D_{1}^{\prime}}, \\
t_{2}=\frac{D^{\prime \prime}}{D^{\prime \prime}-D_{1}^{\prime \prime}}, & 1-t_{2}=\frac{-D_{1}^{\prime \prime}}{D^{\prime \prime}-D_{1}^{\prime \prime}} .
\end{array}
$$

From these values it follows that the cross-ratio of the points $M$ and $M_{1}$ on $V$ and $V_{1}$ and the foci $F_{1}$ and $F_{2}$ of their joins is

$$
R\left(M M_{1}, F_{1} F_{2}\right)=-\frac{D D_{1}^{\prime \prime}}{D^{\prime \prime} D_{1}} \text {. }
$$

Substituting the values (32) in (19), we find that the coördinates of the focal points are of the form

$$
\frac{x D_{1}+x_{1} D}{D_{1}+D} \text {, etc.; } \frac{x D_{1}^{\prime \prime}-x_{1} D^{\prime \prime}}{D_{1}^{\prime \prime}-D^{\prime \prime}} \text {, etc. }
$$

Hence we have

Theorem III. The focal points of the lines joining corresponding points $M$ and $M_{1}$ on the surfaces $V$ and $V_{1}$ divide the segment $M M_{1}$ in the ratios $D / D_{1}$ and $-D^{\prime \prime} / D_{1}^{\prime \prime}$.

\section{Special Surfaces of Voss}

In the preceding discussion we have ignored the possibility that the joins of corresponding points on $V$ and $V_{1}$ may pass through a point, in which case any ruled surface of the congruence is developable and Theorem I loses part of its significance. It is our purpose now to consider this possibility. We shall find that every surface $V$ does not admit of such a transformation. We use $w$ to denote the function $W$ for a surface $V$ which possesses this property.

If we take the origin for the point of concurrence of all the rays, we must have

$$
x+t\left(x_{1}-x\right)=0, \quad y+t\left(y_{1}-y\right)=0, \quad z+t\left(z_{1}-z\right)=0 .
$$

When the expressions from (5) and (23) are substituted in these equations, we obtain three conditions of the form

$$
L X+M X_{1}+N X_{2}=0,
$$

which coexist only in case $L=M=N=0$. This gives the three equations of condition

$$
w+A t=0, \quad \frac{\partial w}{\partial u}-\frac{\partial w}{\partial v}+2 \sin \omega t B=0,
$$

$$
\frac{\partial w}{\partial u}+\frac{\partial w}{\partial v}+2 \cos \omega t C=0 \text {. }
$$


If we solve the last two equations for $\partial w / \partial u$ and $\partial w / \partial v$ and substitute the values of $B$ and $C$ from (27), we have

$\frac{\partial w}{\partial u}+t A \frac{(1+\cos \sigma)}{\sin \sigma} \cos (\theta+\omega)=0, \quad \frac{\partial w}{\partial v}+t A \frac{\cos \sigma-1}{\sin \sigma} \cos (\theta-\omega)$.

Hence equations (35) may be replaced by the first of them and

$$
\frac{\partial \log w}{\partial u}=\frac{1+\cos \sigma}{\sin \sigma} \cos (\theta+\omega), \quad \frac{\partial \log w}{\partial v}=\frac{\cos \sigma-1}{\sin \sigma} \cos (\theta-\omega) .
$$

From the equations (25) for the determination of $w_{1}$ it follows that

$$
w w_{1}=\text { const. }
$$

Hence each solution $\theta$ of equations (10) leads by a quadrature to a surface $V$, which we call a special surface of Voss and denote by $V(\theta, \sigma)$, such that there is another surface of the same kind so related that the lines joining corresponding points of the two surfaces are concurrent. We observe that the relation between these surfaces is reciprocal, and consequently we say that each is the adjoint of the other.

It is evident that if there is applied to a special surface $V(\theta, \sigma)$ a transformation involving a different $\theta$, the relation between the two surfaces will be that of Theorem I.

When the values (36) are substituted in equations (7), we find that the expressions for the second fundamental coefficients $\Delta, \Delta^{\prime \prime}$ of $V(\theta, \sigma)$ are reducible to

$$
\begin{aligned}
\Delta & =\frac{2 w}{\sin \sigma}\left(\frac{2 \cos (\theta-\omega)}{\sin 2 \omega} \frac{\partial \omega}{\partial u}-\frac{1+\cos \sigma}{\sin \sigma}\right) \\
\Delta^{\prime \prime} & =-\frac{2 w}{\sin \sigma}\left(\frac{2 \cos (\theta+\omega)}{\sin 2 \omega} \frac{\partial \omega}{\partial v}+\frac{1-\cos \sigma}{\sin \sigma}\right) .
\end{aligned}
$$

From (26) it follows that the coefficients $\Delta_{1}, \Delta_{1}^{\prime \prime}$ of the adjoint surface are given by

$$
\Delta_{1}=-\Delta\left(1+\frac{A}{w}\right), \quad \Delta_{1}^{\prime \prime}=\Delta^{\prime \prime}\left(1+\frac{A}{w}\right)
$$

Since we have

$$
\Delta_{1} \Delta^{\prime \prime}+\Delta_{1}^{\prime \prime} \Delta=0,
$$

it follows that the asymptotic lines on a special surface of Voss correspond to a conjugate system on its adjoint.

We consider the converse problem, that is, we suppose that $D, D^{\prime \prime}, D_{1}, D_{1}^{\prime \prime}$ satisfy an equation of the form (40). From (26) it follows that either $A=0$, 
or there exists a function $\lambda$ such that

$$
\begin{aligned}
D & =\frac{\lambda}{\sin 2 \omega}\left(2 \cos (\theta-\omega) \frac{\partial \omega}{\partial u}-\frac{1+\cos \sigma}{\sin \sigma} \sin 2 \omega\right), \\
D^{\prime \prime} & =-\frac{\lambda}{\sin 2 \omega}\left(2 \cos (\theta+\omega) \frac{\partial \omega}{\partial v}+\frac{1-\cos \sigma}{\sin \sigma} \sin 2 \omega\right) .
\end{aligned}
$$

If we take $A=0$ and substitute in (25) the value of $W_{1}$ so determined, we find that $D=D^{\prime \prime}=0$, which evidently is impossible.

When the values (41) are substituted in the Codazzi equations (8), we find

$$
\lambda=c u .
$$

Hence $V$ is a special surface of Voss, and we have

Theorem IV. A necessary and sufficient condition that a surface of Voss be "special" is that it admit a transformation $\Omega$ such that the asymptotic lines of the given surface $V$ correspond to a conjugate system on the surface $V_{1}$.

\section{Equations of a Transformation $\Omega(\theta, \sigma)$ in Another Form}

In consequence of equations (36) we may put the fundamental equations (25) in the form

$$
\begin{aligned}
& \frac{\partial}{\partial u}\left(W_{1} w\right)=-w \frac{\partial W}{\partial u}+\frac{\partial w}{\partial u} W \\
& \frac{\partial}{\partial v}\left(W_{1} w\right)=w \frac{\partial W}{\partial v}-\frac{\partial w}{\partial v} W .
\end{aligned}
$$

This shows that the transformation $\Omega(\theta, \sigma)$ is of the general class of transformations of Moutard of equations with equal invariants.* It is a wellknown fact that three linearly independent solutions $\nu_{1}, \nu_{2}, \nu_{3}$ of an equation of the form

$$
\frac{\partial^{2} \theta}{\partial u \partial v}=M \theta
$$

determine a surface $\Sigma$ referred to its asymptotic lines; its coördinates $\xi, \eta, \zeta$ are given by the formulas of Lelieuvre $\dagger$

$$
\frac{\partial \xi}{\partial u}=\left|\begin{array}{cc}
\nu_{2} & \nu_{3} \\
\frac{\partial \nu_{2}}{\partial u} & \frac{\partial \nu_{3}}{\partial v}
\end{array}\right|, \quad \frac{\partial \xi}{\partial v}=-\left|\begin{array}{cc}
\nu_{2} & \nu_{3} \\
\frac{\partial \nu_{2}}{\partial v} & \frac{\partial \nu_{3}}{\partial v}
\end{array}\right| .
$$

If a fourth solution of equation (43) is known, say $\theta_{1}$, the functions $\bar{\nu}_{i}$, defined

* Bianchi, Lezioni di geometria differenziale, vol. 2, p. 47: also Darboux, Leçons sur la théorie générale des surfaces, vol. 2, p. 145.

† E., p. 193. 
by the quadratures

$$
\frac{\partial}{\partial u}\left(\theta_{1} \bar{\nu}_{i}\right)=-\left|\begin{array}{ll}
\theta_{1} & \nu_{i} \\
\frac{\partial \theta_{1}}{\partial u} & \frac{\partial \nu_{i}}{\partial u}
\end{array}\right|, \quad \frac{\partial}{\partial v}\left(\theta_{1} \bar{\nu}_{i}\right)=\left|\begin{array}{ll}
\theta_{1} & \nu_{i} \\
\frac{\partial \theta_{1}}{\partial v} & \frac{\partial \nu_{i}}{\partial v}
\end{array}\right| \quad(i=1,2,3)
$$

satisfy the differential equation

$$
\frac{\partial^{2} \theta}{\partial u \partial v}=\theta_{1} \frac{\partial^{2}}{\partial u \partial v} \frac{1}{\theta_{1}} \cdot \theta
$$

Moreover, the surface $\Sigma_{1}$, determined by equations in $\bar{\nu}_{i}$ analogous to (44), can be so placed in space that $\Sigma$ and $\Sigma_{1}$ are the focal surfaces of a $W$-congruence.*

Since the total curvature of $\Sigma$ is given by $\dagger$

$$
K=\frac{-1}{\left(\nu_{1}^{2}+\nu_{2}^{2}+\nu_{3}^{2}\right)^{2}},
$$

if $\Sigma$ and $\Sigma_{1}$ are to be pseudospherical surfaces of curvature $-\left(1 / a^{2}\right)$ in the relation of a transformation of Bäcklund,

$$
\begin{aligned}
& \nu_{1}=\sqrt{a} X, \quad \nu_{2}=\sqrt{a} \bar{Y}, \quad \nu_{3}=\sqrt{a} Z, \\
& \bar{\nu}_{1}=\sqrt{a} X^{\prime}, \quad \bar{\nu}_{2}=\sqrt{a} Y^{\prime}, \quad \bar{\nu}_{3}=\sqrt{a} Z^{\prime},
\end{aligned}
$$

where $X^{\prime}, Y^{\prime}, Z^{\prime}$ are given by (11). When these values, are substituted in (45), we find that $\theta_{1}$ equals $w$, given by (36), to within a constant factor. Now, equations (43) and (43') are respectively (4) and (17). Hence we have

Theorem V. A transformation $\Omega(\theta, \sigma)$ is a transformation of Moutard in which the transforming function is the tangential coordinate $w$ of the corresponding special surface $V(\theta, \sigma)$.

It is a fundamental property of $W$-congruences that each focal surface admits an infinitesimal deformation parallel to the normal to the other surface. The surface corresponding with orthogonality of linear elements to $\Sigma$, defined by (44), in this case has for coördinates $\ddagger$

$$
\theta_{1} \bar{\nu}_{1}, \quad \theta_{1} \bar{\nu}_{2}, \quad \theta_{1} \nu_{8} .
$$

Moreover, in the general case $\theta_{1} V=K$ is the tangential coördinate $W$ of the corresponding associate surface. Hence we have

Theorem VI. A special surface $V(\theta, \sigma)$ associated with a pseudospherical surface $P$ is that associate surface in the infinitesimal deformation of $P$ whose directrices are parallel to the corresponding normals to the Bäcklund transform of $P$ by means of $\theta$ and $\sigma$.

E., pp. 418, 419.

† E., p. 194.

$\ddagger$ E., p. 420. 
Since $W_{1} w$ is determined by (42) only to within an additive constant, and since also $1 / w$ is a solution of equation (17), if $V_{1}$ is a transform of $V$ by a transformation $\Omega(\theta, \sigma)$, so also is the suite of surfaces whose tangential coördinates are

$$
X^{\prime}, \quad Y^{\prime}, \quad Z^{\prime}, \quad W_{1}+\frac{a}{w},
$$

where $a$ is an arbitrary constant.

In like manner it may be observed that $V_{1}$ is obtained by a transformation $\Omega(\theta, \sigma)$ from any one of the surfaces whose tangential coördinates are

$$
X, Y, Z, W+a w,
$$

provided it is true when $a=0$.

We have seen that when a surface $V(\theta, \sigma)$ is transformed by $\Omega(\theta, \sigma)$, the tangential coördinates $w$ and $w_{1}$ are such that their product is constant. We shall show that this property is characteristic of special surfaces. In fact, if we differentiate the equation

$$
W W_{1}=\text { const. }
$$

with respect to $u$ and $v$ respectively and make use of equations (42), we get

$$
\left(W_{1}-W\right) \frac{\partial}{\partial u} \log \frac{W}{w}=0, \quad\left(W_{1}-W\right) \frac{\partial}{\partial v} \log \frac{W}{w}=0 .
$$

Hence $V$ is a special surface $V(\theta, \sigma)$ and the transformation is $\Omega(\theta, \sigma)$. We shall say that in this case the two special surfaces are in perspective correspondence.

\section{Theorem of Permutabiuty of the Transformations $\Omega$}

It is our purpose to show that the transformations which have been established in $\$ 2$ admit a " theorem of permutability " as follows:

Theorem VII. If two surfaces $V_{1}$ and $V_{2}$ are obtained from a surface $V$ by means of transformations $\Omega\left(\theta_{1}, \sigma_{1}\right)$ and $\Omega\left(\theta_{2}, \sigma_{2}\right)$ respectively; there exists $a$ surface $V^{\prime}$ which may be obtained from $V_{1}$ by a transformation $\Omega\left(\phi, \sigma_{2}\right)$ and from $V_{2}$ by a transformation $\Omega\left(\phi, \sigma_{1}\right)$; moreover, the determination of $V^{\prime}$ does not involve integration.

If $W_{1}$ and $W_{2}$ are tangential coördinates of $V_{1}$ and $V_{2}$ respectively, we must have in accordance with (42)

$$
\begin{aligned}
& \frac{\partial W_{i}}{\partial u}+\frac{W_{i}}{w_{i}} \frac{\partial w_{i}}{\partial u}=-\frac{\partial W}{\partial u}+\frac{W}{w_{i}} \frac{\partial w_{i}}{\partial u}, \\
& \frac{\partial W_{i}}{\partial v}+\frac{W_{i}}{w_{i}} \frac{\partial w_{i}}{\partial v}=\frac{\partial W}{\partial v}-\frac{W}{w_{i}} \frac{\partial w_{i}}{\partial v}
\end{aligned}
$$$$
(i=1,2) \text {, }
$$ 
where the functions $w_{1}$ and $w_{2}$ are given by

and

$$
\begin{aligned}
& \frac{\partial \log w_{i}}{\partial u}=\frac{1+\cos \sigma_{i}}{\sin \sigma_{i}} \cos \left(\theta_{i}+\omega\right), \\
& \frac{\partial \log w_{i}}{\partial v}=\frac{\cos \sigma_{i}-1}{\sin \sigma_{i}} \cos \left(\theta_{i}-\omega\right)
\end{aligned}
$$

$$
\begin{aligned}
& \sin \sigma_{i}\left(\frac{\partial \theta_{i}}{\partial u}-\frac{\partial \omega}{\partial u}\right)+\left(\cos \sigma_{i}+1\right) \sin \left(\theta_{i}+\omega\right)=0, \\
& \sin \sigma_{i}\left(\frac{\partial \theta_{i}}{\partial v}+\frac{\partial \omega}{\partial v}\right)+\left(\cos \sigma_{i}-1\right) \sin \left(\theta_{i}-\omega\right)=0
\end{aligned}
$$

On the assumption that a surface $V^{\prime}$ exists satisfying the requirements of the above theorem, there must exist functions $W^{\prime}, w_{1}^{\prime}$, and $w_{2}^{\prime}$ satisfying equations analogous to (46), namely

$$
\begin{aligned}
& \frac{\partial W^{\prime}}{\partial u}+\frac{W^{\prime}}{w_{i}^{\prime}} \frac{\partial w_{i}^{\prime}}{\partial u}=-\frac{\partial W_{i}}{\partial u}+\frac{W_{i}}{w_{i}^{\prime}} \frac{\partial w_{i}^{\prime}}{\partial u}, \\
& \frac{\partial W^{\prime}}{\partial v}+\frac{W^{\prime}}{w_{i}^{\prime}} \frac{\partial w_{i}^{\prime}}{\partial v}=\frac{\partial W_{i}}{\partial v}-\frac{W_{i}}{w_{i}^{\prime}} \frac{\partial w_{i}^{\prime}}{\partial v}
\end{aligned}
$$

By means of (46) these are reducible to

$$
\begin{aligned}
& \frac{\partial W^{\prime}}{\partial u}+W^{\prime} \frac{\partial \log w_{i}^{\prime}}{\partial u}=W_{i} \cdot \frac{\partial}{\partial u} \log \left(w_{i}^{\prime} w_{i}\right)+\frac{\partial W}{\partial u}-W \frac{\partial \log w_{i}}{\partial u}, \\
& \frac{\partial W^{\prime}}{\partial v}+W^{\prime} \frac{\partial \log w_{i}^{\prime}}{\partial v}=-W_{i} \cdot \frac{\partial}{\partial v} \log \left(w_{i}^{\prime} w_{i}\right)+\frac{\partial W}{\partial v}-W \frac{\partial \log w_{i}}{\partial v} \\
& (i=1,2) .
\end{aligned}
$$

The consistency of the first equations for $i=1$ and $i=2$, and likewise for the second equations, requires that

$$
\begin{aligned}
W^{\prime} \frac{\partial}{\partial u} \log \frac{w_{1}^{\prime}}{w_{2}^{\prime}}-W_{1} \frac{\partial}{\partial u} \log \left(w_{1}^{\prime} w_{1}\right) & \\
& +W_{2} \frac{\partial}{\partial u} \log \left(w_{2}^{\prime} w_{2}\right)+W \frac{\partial}{\partial u} \log \frac{w_{1}}{w_{2}}=0, \\
W^{\prime} \frac{\partial}{\partial v} \log \frac{w_{1}^{\prime}}{w_{2}^{\prime}}+W_{1} \frac{\partial}{\partial v} \log \left(w_{1}^{\prime} w_{1}\right) & -W_{2} \frac{\partial}{\partial v} \log \left(w_{2}^{\prime} w_{2}\right)+W \frac{\partial}{\partial v} \log \frac{w_{1}}{w_{2}}=0 .
\end{aligned}
$$

Since $V_{1}$ and $V_{2}$ are general transforms of $V$, the coexistence of equations 
(51) necessitates the conditions

$$
\begin{gathered}
\frac{\partial}{\partial v} \log \frac{w_{1}^{\prime}}{w_{2}^{\prime}} \frac{\partial}{\partial u} \log w_{1}^{\prime} w_{1}+\frac{\partial}{\partial u} \log \frac{w_{1}^{\prime}}{w_{2}^{\prime}} \frac{\partial}{\partial v} \log w_{1}^{\prime} w_{1}=0, \\
\frac{\partial}{\partial v} \log \frac{w_{1}^{\prime}}{w_{2}^{\prime}} \frac{\partial}{\partial u} \log w_{2}^{\prime} w_{2}+\frac{\partial}{\partial u} \log \frac{w_{1}^{\prime}}{w_{2}^{\prime}} \frac{\partial}{\partial v} \log w_{2}^{\prime} w_{2}=0, . \\
\frac{\partial}{\partial v} \log \frac{w_{1}^{\prime}}{w_{2}^{\prime}} \frac{\partial}{\partial u} \log \frac{w_{2}}{w_{1}}-\frac{\partial}{\partial u} \log \frac{w_{1}^{\prime}}{w_{2}^{\prime}} \frac{\partial}{\partial v} \log \frac{w_{2}}{w_{1}}=0,
\end{gathered}
$$

This system may be replaced by the first and the following equations

$$
\begin{aligned}
& \frac{\partial}{\partial u}\left(w_{1}^{\prime} w_{1}\right)=-\mu\left(w_{1} \frac{\partial w_{2}}{\partial u}-w_{2} \frac{\partial w_{1}}{\partial u}\right), \\
& \frac{\partial}{\partial u}\left(w_{2}^{\prime} w_{2}\right)=+\lambda\left(w_{1} \frac{\partial w_{2}}{\partial u}-w_{2} \frac{\partial w_{1}}{\partial u}\right), \\
& \frac{\partial}{\partial v}\left(w_{1}^{\prime} w_{1}\right)=\mu\left(w_{1} \frac{\partial w_{2}}{\partial v}-w_{2} \frac{\partial w_{1}}{\partial v}\right), \\
& \frac{\partial}{\partial v}\left(w_{2}^{\prime} w_{2}\right)=-\lambda\left(w_{1} \frac{\partial w_{2}}{\partial v}-w_{2} \frac{\partial w_{1}}{\partial v}\right),
\end{aligned}
$$

where $\lambda$ and $\mu$ are functions to be determined. When these values are substituted in the first of equations (52) we find that it may be replaced by

$$
\mu w_{2} w_{2}^{\prime}+\lambda w_{1} w_{1}^{\prime}=0 \text {. }
$$

In consequence of (53) and (54) equations (51) reduce to

$$
W^{\prime}=\left(W_{1}-W_{2}\right) \lambda \frac{w_{1}}{w_{2}^{\prime}}+W=\left(W_{2}-W_{1}\right) \mu \frac{w_{2}}{w_{1}^{\prime}}+W \text {. }
$$

If we substitute these expressions in (50), we find that $\lambda$ and $\mu$ must be constant.

It is necessary now that we determine whether functions $w_{2}^{\prime}$ and $w_{1}^{\prime}$ exist which satisfy equations (53) and also equations analogous to (47), namely

$$
\begin{aligned}
& \frac{\partial \log w_{i}^{\prime}}{\partial u}=\frac{1+\cos \sigma_{k}}{\sin \sigma_{k}} \cos \left(\phi+\theta_{i}\right), \\
& \frac{\partial \log w_{i}^{\prime}}{\partial v}=\frac{\cos \sigma_{k}-1}{\sin \sigma_{k}} \cos \left(\phi-\theta_{i}\right)
\end{aligned} \quad(i, k=1,2 ; i \neq k),
$$

where the function $\phi$ must satisfy the equations

$$
\begin{aligned}
& \sin \sigma_{i}\left(\frac{\partial \phi}{\partial u}-\frac{\partial \theta_{k}}{\partial u}\right)+\left(\cos \sigma_{i}+1\right) \sin \left(\phi+\theta_{k}\right)=0, \\
& \sin \sigma_{i}\left(\frac{\partial \phi}{\partial v}+\frac{\partial \theta_{k}}{\partial v}\right)+\left(\cos \sigma_{i}-1\right) \sin \left(\phi-\theta_{k}\right)=0
\end{aligned}
$$


From the "theorem of permutability" of transformations of Bäcklund of pseudospherical surfaces it follows that there exists a function $\phi$ satisfying the four equations (57); it is given by *

$$
\begin{aligned}
& \sin (\phi-\omega)=\frac{\left(\cos \sigma_{1}-\cos \sigma_{2}\right) \sin \left(\theta_{2}-\theta_{1}\right)}{\sin \sigma_{1} \sin \sigma_{2} \cos \left(\theta_{2}-\theta_{1}\right)+\cos \sigma_{1} \cos \sigma_{2}-1} \\
& \cos (\phi-\omega)=\frac{\sin \sigma_{1} \sin \sigma_{2}+\left(\cos \sigma_{1} \cos \sigma_{2}-1\right) \cos \left(\theta_{2}-\theta_{1}\right)}{\sin \sigma_{1} \sin \sigma_{2} \cos \left(\theta_{2}-\theta_{1}\right)+\cos \sigma_{1} \cos \sigma_{2}-1} .
\end{aligned}
$$

Since $w_{1}^{\prime}$ and $w_{2}^{\prime}$ are given by (56) only to within a constant factor, it follows from (53) that the constants $\mu$ and $\lambda$ may be taken equal to unity, and consequently equations (53) may be written

$$
\begin{aligned}
& \frac{\partial}{\partial u}\left(w_{i}^{\prime} w_{i}\right)=w_{k} \frac{\partial w_{i}}{\partial u}-w_{i} \frac{\partial w_{k}}{\partial u}, \\
& \frac{\partial}{\partial v}\left(w_{i}^{\prime} w_{i}\right)=-w_{k} \frac{\partial w_{i}}{\partial v}+w_{i} \frac{\partial w_{k}}{\partial v}
\end{aligned}
$$

If we reduce these equations for $i=1, k=2$ by means of (56) and (47), we obtain

$$
\begin{aligned}
& w_{1}^{\prime}\left(\frac{1+\cos \sigma_{2}}{\sin \sigma_{2}} \cos \left(\phi+\theta_{1}\right)+\frac{1+\cos \sigma_{1}}{\sin \sigma_{1}} \cos \left(\theta_{1}+\omega\right)\right) \\
& \quad=-w_{2}\left(\frac{1+\cos \sigma_{2}}{\sin \sigma_{2}} \cos \left(\theta_{2}+\omega\right)-\frac{1+\cos \sigma_{1}}{\sin \sigma_{1}} \cos \left(\theta_{1}+\omega\right)\right),
\end{aligned}
$$

$$
\begin{aligned}
& w_{1}^{\prime}\left(\frac{\cos \sigma_{2}-1}{\sin \sigma_{2}} \cos \left(\phi-\theta_{1}\right)+\frac{\cos \sigma_{1}-1}{\sin \sigma_{1}} \cos \left(\theta_{1}-\omega\right)\right) \\
& =w_{2}\left(\frac{\cos \sigma_{2}-1}{\sin \sigma_{2}} \cos \left(\theta_{2}-\omega\right)-\frac{\cos \sigma_{1}-1}{\sin \sigma_{1}} \cos \left(\theta_{1}-\omega\right)\right) .
\end{aligned}
$$

If we add these equations, the result is reducible to

$$
\begin{aligned}
& \frac{w_{1}^{\prime}}{w_{2}}\left[\left(\cot \sigma_{2} \cos \phi+\cot \sigma_{1} \cos \omega\right) \cos \theta_{1}\right. \\
& \left.\quad-\left(\frac{1}{\sin \sigma_{2}} \sin \phi+\frac{1}{\sin \sigma_{1}} \sin \omega\right) \sin \theta_{1}\right] \\
& \quad=\left(-\frac{\cos \theta_{2}}{\sin \sigma_{2}}+\frac{\cos \theta_{1}}{\sin \sigma_{1}}\right) \cos \omega+\left(\cot \sigma_{2} \sin \theta_{2}-\cot \sigma_{1} \sin \theta_{1}\right) \sin \omega .
\end{aligned}
$$

By means of the values of $\phi$ given by (58) this equation becomes

$$
\frac{w_{1}^{\prime}}{w_{2}}=\frac{\sin \sigma_{1} \sin \sigma_{2} \cos \left(\theta_{2}-\theta_{1}\right)+\cos \sigma_{1} \cos \sigma_{2}-1}{\cos \sigma_{2}-\cos \sigma_{1}} .
$$

\footnotetext{
*E., p. 287.
} 
The same result is obtained if equations (60) be subtracted from each other and in the resulting equation the values of the functions of $\phi$ given by (58) be substituted. It is readily shown with the aid of (58) that this expression for $w_{1}^{\prime}$ satisfies equations (56) with $i=1, k=2$.

If we proceed in a similar manner with equations (59) with $i=2, k=1$, we obtain

$$
\frac{w_{2}^{\prime}}{w_{1}}=-\frac{\sin \sigma_{1} \sin \sigma_{2} \cos \left(\theta_{2}-\theta_{1}\right)+\cos \sigma_{1} \cos \sigma_{2}-1}{\cos \sigma_{2}-\cos \sigma_{1}} .
$$

These values of $w^{\prime}$ and $w_{2}^{\prime}$ are in agreement with (54) which now is

$$
w_{2}^{\prime} w_{2}+w_{1}^{\prime} w_{1}=0 \text {. }
$$

From (55) it follows that the tangential coördinate $W^{\prime}$ of the surface $V$ is

$$
W^{\prime}=W+\frac{\left(W_{2}-W_{1}\right)\left(\cos \sigma_{2}-\cos \sigma_{1}\right)}{\sin \sigma_{1} \sin \sigma_{2} \cos \left(\theta_{2}-\theta_{1}\right)+\cos \sigma_{1} \cos \sigma_{2}-1} .
$$

Hence the "theorem of permutability," as stated at the beginning of this section, has been completely established.

Suppose that $W=w_{1}$ and $W_{1}=1 / w_{1}$; it is evident that the character of $W_{2}$ and $W^{\prime}$ depend upon the choice of the function $w_{2}$. If we require that $V_{2}$ and $V^{\prime}$ shall be special surfaces of Voss such that the joins of corresponding points are concurrent, in all generality we can put $W^{\prime} W_{2}=1$. It follows at once from (65) and (63) that $W_{2}=w_{2}^{\prime}$. Now $V_{1}$ and $V^{\prime}$ are not in perspective correspondence. Otherwise we should have $w_{1}^{\prime} w_{1}=$ const., which is inconsistent with (59).

If four surfaces are related in accordance with Theorem VII, we say that they form a quatern. In view of the above results we have

TheOREM VIII. If $V, V_{1}, V_{2}, V^{\prime}$ form a quatern of surfaces of $V$ oss determined by a set of functions $w_{1}, w_{2}, w_{1}^{\prime}, w_{2}^{\prime}$, the special surfaces of Voss determined by the functions $w_{1}, 1 / w_{1}, w_{2}^{\prime}, 1 / w_{2}^{\prime}$ form a quatern, and so likewise do the special surfaces determined by the functions $w_{2}, 1 / \mathfrak{i}_{2}, w_{1}^{\prime}, 1 / w_{1}^{\prime}$.

Conversely,

Theorem IX. If two surfaces $V$ and $V_{1}$ of a quatern $\left(V, V_{1}, V_{2}, V^{\prime}\right)$ are special surfaces in perspective correspondence, the necessary and sufficient condition that $V_{2}$ and $V^{\prime}$ be special surfaces in perspective correspondence is that these surfaces be determined by $w_{2}^{\prime}$ and $1 / w_{2}^{\prime}$, where $w_{2}^{\prime}$ is given by (83), $\sigma_{2}$ being any constant and $\theta_{2} a$ solution of the corresponding equations (10).

\section{Normal Congruences Determined by Trangformations $\Omega$}

We turn now to the consideration of the congruence of the lines of intersection of the tangent planes at the corresponding points $M$ and $M_{1}$ on two 
surfaces $V$ and $V_{1}$, of which the latter arises from $V$ by a transformation $\Omega$. From the general theory of congruences* we know that the tangents at $M$ and $M_{1}$ to the geodesics $v=$ const. through these respective points meet in a point $F_{1}$; likewise, the tangents at $M$ and $M_{1}$ to the geodesics $u=$ const. meet in a point $F_{2}$; furthermore, these points $F_{1}$ and $F_{2}$ are the focal points of their join in the congruence formed by these lines of intersection of the tangent planes to $V$ and $V_{1}$ at $M$ and $M_{1}$.

If the coördinates of $F_{1}$ and $F_{2}$ be denoted by $a_{1}, b_{1}, c_{1} ; a_{2}, b_{2}, c_{2}$ respectively,

$$
\begin{aligned}
& a_{1}=x+\lambda_{1}\left(\cos \omega X_{1}+\sin \omega X_{2}\right)=x_{1}+\mu_{1}\left(\cos \theta X_{1}^{\prime}+\sin \theta X_{2}^{\prime}\right), \\
& a_{2}=x+\lambda_{2}\left(\cos \omega X_{1}-\sin \omega X_{2}\right)=x_{1}+\mu_{2}\left(\cos \theta X_{1}^{\prime}-\sin \theta X_{2}^{\prime}\right),
\end{aligned}
$$

where $\lambda_{1}, \mu_{1}, \lambda_{2}, \mu_{2}$ are quantities to be determined. From (16) and (23) it follows that the equivalence of the two expressions for $a_{1}, h_{1}$, and $c_{1}$ requires that $\lambda_{1}$ and $\mu_{1}$ satisfy

$$
\begin{aligned}
& A+\mu_{1} \sin \sigma \sin (\theta-\omega)=0, \\
& B-\mu_{1}[\sin (\theta-\omega) \cos \sigma \sin \theta+\cos (\theta-\omega) \cos \theta]-\lambda_{1} \cos \omega=0, \\
& C+\mu_{1}[\sin (\theta-\omega) \cos \sigma \cos \theta-\cos (\theta-\omega) \sin \theta]-\lambda_{1} \sin \omega=0 .
\end{aligned}
$$

It is found that these equations are consistent in view of the values (27) of $A, B$, and $C$.

From the above equations we find

$$
\lambda_{1}=\frac{A \sin 2 \theta}{\sin \sigma \sin 2 \omega \sin (\theta-\omega)}, \quad \mu_{1}=\frac{-A}{\sin \sigma \sin (\theta-\omega)} .
$$

In like manner it can be shown that

$$
\lambda_{2}=\frac{A \sin 2 \theta}{\sin \sigma \sin 2 \omega \sin (\theta+\omega)}, \quad \mu_{2}=\frac{A}{\sin \sigma \sin (\theta+\omega)} .
$$

We shall need the expressions for $\partial A / \partial u$ and $\partial A / \partial v$. They are most easily obtained indirectly by differentiating equation (23) with respect to $u$ and $v$ separately and equating to zero the coefficients of $X$ in each case. These expressions are reducible to

$$
\begin{aligned}
& \frac{\partial A}{\partial u}=-\frac{D_{1}}{\sin 2 \theta} \sin \sigma \sin (\theta-\omega)+\frac{A(1+\cos \sigma) \cos (\theta+\omega)}{\sin \sigma}, \\
& \frac{\partial A}{\partial v}=\frac{-D_{1}^{\prime \prime}}{\sin 2 \theta} \sin \sigma \sin (\theta+\omega)+\frac{A(\cos \sigma-1) \cos (\theta-\omega)}{\sin \sigma} .
\end{aligned}
$$

If we substitute in (66) the values of $\lambda_{1}$ and $\lambda_{2}$ given above, we derive by * Cf. Darboux, Leģons sur la théorie générale des surfaces, vol. 2, pp. 230, 231. 
means of (69) the following:

$$
\frac{\partial a_{1}}{\partial u}=\frac{\lambda_{1} \sin 2 \omega}{\sin (\theta-\omega)}\left(\frac{\cos \sigma+1}{\sin \sigma}\left(\cos \omega X_{1}+\sin \omega X_{2}\right)-\sin \left(\theta_{0}-\omega\right) X\right),
$$

$$
\frac{\partial a_{1}}{\partial v}=\left(2 \lambda_{1} \frac{\partial \omega}{\partial v}-D^{\prime \prime}\right) \frac{\left(\cos \theta X_{1}+\sin \theta X_{2}\right)}{\sin (\theta-\omega)},
$$

$$
\begin{aligned}
& \frac{\partial a_{2}}{\partial u}=-\left(2 \lambda_{2} \frac{\partial \omega}{\partial u}+D\right) \frac{\left(\cos \theta X_{1}+\sin \theta X_{2}\right)}{\sin (\theta+\omega)} \\
& \frac{\partial a_{2}}{\partial v}=\frac{\lambda_{2} \sin 2 \omega}{\sin (\theta+\omega)}\left(\frac{1-\cos \sigma}{\sin \sigma}\left(\cos \omega X_{1}-\sin \omega X_{2}\right)+\sin (\theta+\omega) X\right) .
\end{aligned}
$$

These equations show incidentally that $F_{1}$ and $F_{2}$ are the focal points of the congruence.

We denote by $\Sigma_{1}$ and $\Sigma_{2}$ the focal surfaces of the congruence, that is the loci of the points $F_{1}$ and $F_{2}$, and by $A_{1}, B_{1}, C_{1}, A_{2}, B_{2}$, and $C_{2}$ the directioncosines of the normals to $\Sigma_{1}$ and $\Sigma_{2}$. From (70) and (71) we find

$$
\begin{aligned}
& A_{1}=\left(\sin \theta X_{1}-\cos \theta X_{2}+\frac{1+\cos \sigma}{\sin \sigma} X\right) \frac{\sin \sigma}{\sqrt{2(1+\cos \sigma)}}, \\
& A_{2}=\left(\sin \theta X_{1}-\cos \theta X_{2}+\frac{\cos \sigma-1}{\sin \sigma} X\right) \frac{\sin \sigma}{\sqrt{2(1-\cos \sigma)}},
\end{aligned}
$$

and similar expressions for the $B$ 's and $C$ 's.

Hence we have

$$
\begin{array}{ll}
\sum X A_{1}=\cos \frac{\sigma}{2}, & \sum X^{\prime} A_{1}=\cos \frac{\sigma}{2}, \\
\sum X A_{2}=-\sin \frac{\sigma}{2}, & \sum X^{\prime} A_{2}=\sin \frac{\sigma}{2} .
\end{array}
$$

Consequently we have

THEOREM X. For the congruence of the lines of intersection of the tangent planes at corresponding points on $V_{1}$ and $V$ the focal planes bisect the angles between the tangent planes, and consequently the congruence is normal.

From (66) it follows that

$$
a_{1}-a_{2}=\frac{A \sin 2 \theta}{\sin \sigma \sin (\theta-\omega) \sin (\theta+\omega)}\left(X_{1} \cos \theta+X_{2} \sin \theta\right),
$$

consequently the focal distance $2 \rho$ is given by

$$
2 \rho=\frac{A \sin 2 \theta}{\sin \sigma \sin (\theta-\omega) \sin (\theta+\omega)} .
$$




\section{Converse Problem}

Suppose that we have a pseudospherical congruence, that is the congruence of lines joining points of a pseudospherical surface $P$ to the corresponding points of a Bäcklund transform $P_{1}$. Using the notation of the preceding sections, we note that the direction-cosines of this congruence, namely $\bar{X}$, $\bar{Y}$, and $\bar{Z}$, are of the form

$$
\bar{X}=\cos \theta X_{1}+\sin \theta X_{2} .
$$

By differentiation we obtain

$$
\begin{aligned}
& \frac{\partial \bar{X}}{\partial u}=\sin (\theta+\omega)\left[\left(\sin \theta X_{1}-\cos \theta X_{2}\right) \frac{\cos \sigma+1}{\sin \sigma}-X\right], \\
& \frac{\partial \bar{X}}{\partial v}=\sin (\theta-\omega)\left[\left(\sin \theta X_{1}-\cos \theta X_{2}\right) \frac{\cos \sigma-1}{\sin \sigma}-X\right] .
\end{aligned}
$$

From these expressions we derive the following expressions for the coefficients of the linear element of the spherical representation of this congruence:

$$
\sqrt{\mathcal{S}}=\frac{\sin (\theta+\omega)}{\sin \frac{1}{2} \sigma}, \quad \mathscr{F}=0, \quad \sqrt{\mathcal{S}}=\frac{\sin (\theta-\omega)}{\cos \frac{1}{2} \sigma} .
$$

It must be remembered that the parametric curves on the unit sphere correspond to the asymptotic lines on the focal surfaces of the congruence. Since this system on the sphere is orthogonal, it represents the lines of curvature of a group of surfaces $\bar{S}$ whose fundamental functions $\bar{D}$ and $\bar{D}^{\prime \prime}$ satisfy the Codazzi equations

$$
\frac{\partial}{\partial v}\left(\frac{\bar{D}}{\sqrt{\mathcal{S}}}\right)=\frac{\bar{D}^{\prime \prime}}{\mathcal{G}} \frac{\partial \sqrt{\mathcal{S}}}{\partial v}, \quad \frac{\partial}{\partial u}\left(\frac{\bar{D}^{\prime \prime}}{\sqrt{\mathcal{G}}}\right)=\frac{\bar{D}}{\mathcal{S}} \frac{\partial \sqrt{\mathcal{G}}}{\partial u}
$$

Each set of functions $\bar{D}, \bar{D}^{\prime \prime}$ satisfying these equations gives a surface $\bar{S}$ by means of the quadratures *

$$
\frac{\partial \bar{x}}{\partial u}=-\frac{\bar{D} \partial \bar{X}}{\mathcal{S} \partial u}, \quad \frac{\partial \bar{x}}{\partial v}=-\frac{\bar{D}^{\prime \prime}}{\mathcal{G}} \frac{\partial \bar{X}}{\partial v} .
$$

Suppose we have such a surface $\bar{S}$. Through each normal we draw planes parallel to the corresponding tangent planes to the pseudospherical surfaces $P$ and $P_{1}$. The tangential coördinates $W$ and $W_{1}$ of the envelopes of these planes are given by

* E., p. 161.

$$
W=\sum \bar{x} X, \quad W_{1}=\sum \bar{x} X^{\prime} .
$$


With the aid of (79) and (80) we show that

$$
\frac{\partial^{2} W}{\partial u \partial v}+\cos 2 \omega W=0, \quad \frac{\partial^{2} W_{1}}{\partial u \partial v}+\cos 2 \theta W_{1}=0 .
$$

Hence the envelopes of these planes are surfaces of Voss, and we have

Theorem XI. If $P$ and $P_{1}$ are any two pseudospherical surfaces in the relation of a Bäcklund transformation, there exists a family of surfaces $\bar{S}$ each of which has its normals parallel to lines joining corresponding points of $P$ and $P_{1}$, and, in this correspondence between the surfaces $\bar{S}, P$, and $P_{1}$, the lines of curvature on the first correspond to the asymptotic lines on the last two surfaces. Moreover, the two planes through each normal to $\bar{S}$ and parallel to the tangent planes to $P$ and $P_{1}$ at the corresponding points envelope two surfaces of Voss which are in the relation of a transformation $\Omega$.

Princeron,

January 12, 1914. 